\title{
VALORISATION ET DEVELOPPEMENT TOURISTIQUE DE LA "GROTTA DI BOSSEA" (Frabosa Soprana, Cuneo, Italie)
}

\author{
Guido Peano* \& Giorgio Fisanotti**
}

\begin{abstract}
The exploitation and devopment of the "Grotta di Bossea" as a show cave]
\end{abstract}
The Bossea Cave has a great interest from the point of view of nature and the environment because many karst and speleogenetic processes are still active. The water stream flowing in the cave was the determining factor of some picturesque morphologies and splendid scenographic effects.

An underground laboratory for experimental researches (the "Bossea Scientific Station") was established in the cave and is operating since 1969 (Biological Section) and 1982 (Physical Section).

The cave has been explored and studied since 1850 and opened to the public in 1874. At present its exploitation can be furtherly emphasized.

For this purpose a programme was prepared according the following items:

1 - rearrangement of the tourist facilities

2 - best exploitation of internal hydrography

3 - creation of alternative trails

4 - improvement of the scientific facilities

5 - creation of new outside facilities

6 - preparation of literature and visual aids.

The goals of the Scientific Station installed inside the cave and the results achieved, are described by Guido Peano, laboratory director; the cave exploitation project, concerning both the scientific and tourist facilities, is due to Giorgio Fisanotti.

\section{RIASSUNTO [Lo sviluppo turistico della "Grotta di Bossea" ]}

La grotta di Bossea è particolarmente interessante dal punto di vista della natura e dell'ambiente poiché molti processi carsici e speleogenetici sono tuttora attivi. Il corso d'acqua che la attraversa è il fattore determinante per varie morfologie pittoresche e per splendidi effetti scenografici.

Un laboratorio sotterraneo per ricerche sperimentali (la "Stazione Scientifica di Bossea") è stato installato nella grotta; la Sezione biologica è operativa dal 1969 mentre quella fisica lo è dal 1980 .

La grotta è stata esplorata e studiata fin dal 1850 ed è stata aperta al pubblico nel 1874 . Attualmente il suo sviluppo turistico puo essere ulteriormente incrementato. A tal fine èstato elaborato un programma così articolato:

1 - rifacimento delle attrezzature turistiche

2 - miglior sfruttamento dell'idrografia sotterranea

3 - creazione di percorsi alternativi

4 - miglioramento della strumentazione scientifica

5 - creazione di nuove attrezzature all'esterno

6 - preparazione di pubblicazioni ed audiovisivi

Gli scopi della Stazione Scientifica installata in grotta ed i risultati raggiunti sono stati descritti da Guido Peano, direttore del laboratorio; il progetto di sfruttamento della grotta, riguardante sia le attrezzature scientifiche che quelle turistiche è opera di Giorgio Fisanotti.

\footnotetext{
* Via Bassignano 5, I-12100 CUNEO (Italie)

** Via Gottardo 167, I- 10154 TORINO (Italie)
} 
GROTTA DI BOSSEA (108Pi)

G.S.A.M. C.A.I. Cuneo 1988

Calteris, Chesta, Elia
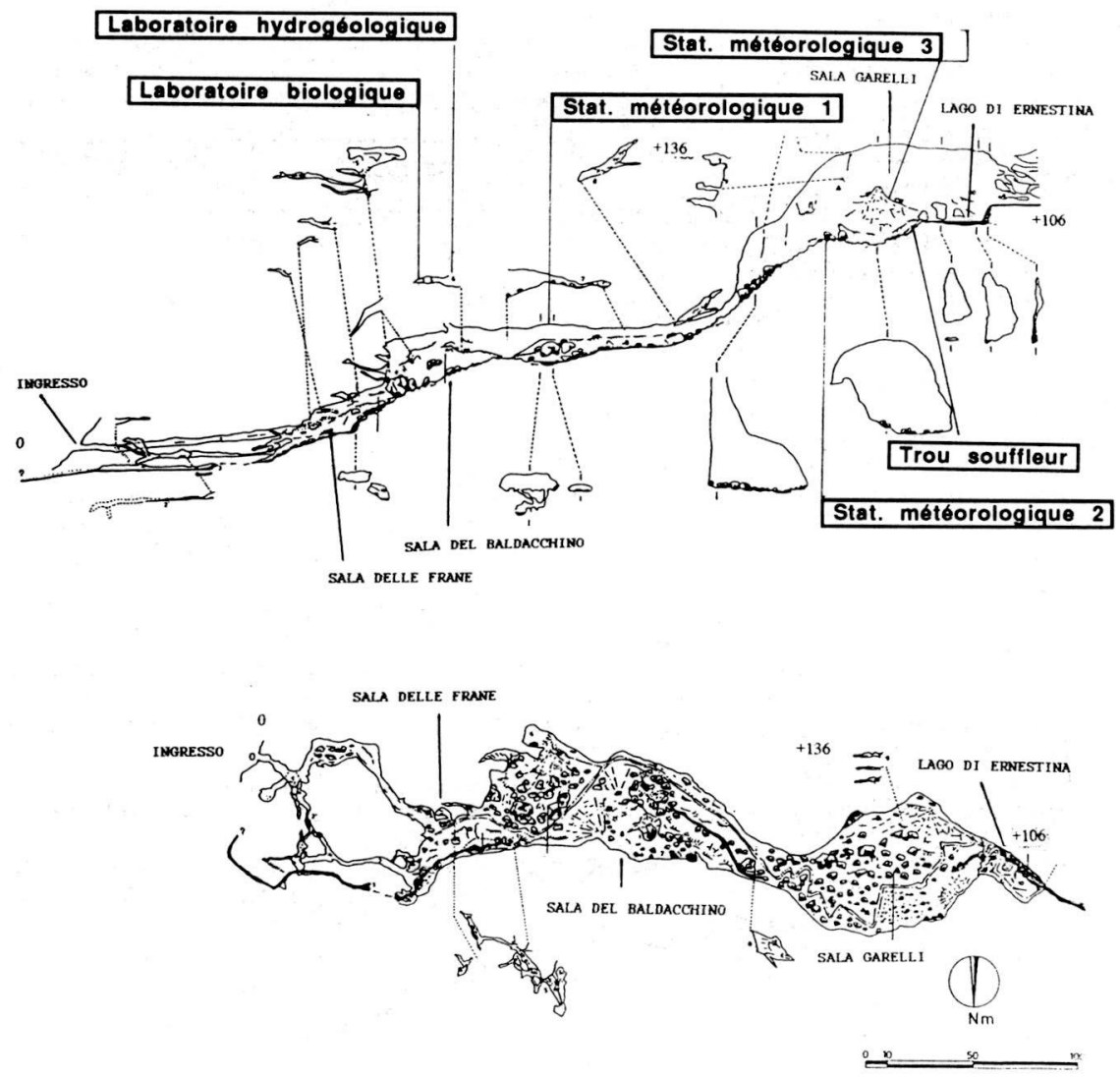

Lieu de relevé de données

Fig. 1 - Plan de la "Grotta di Bossea": zone inférieure. 

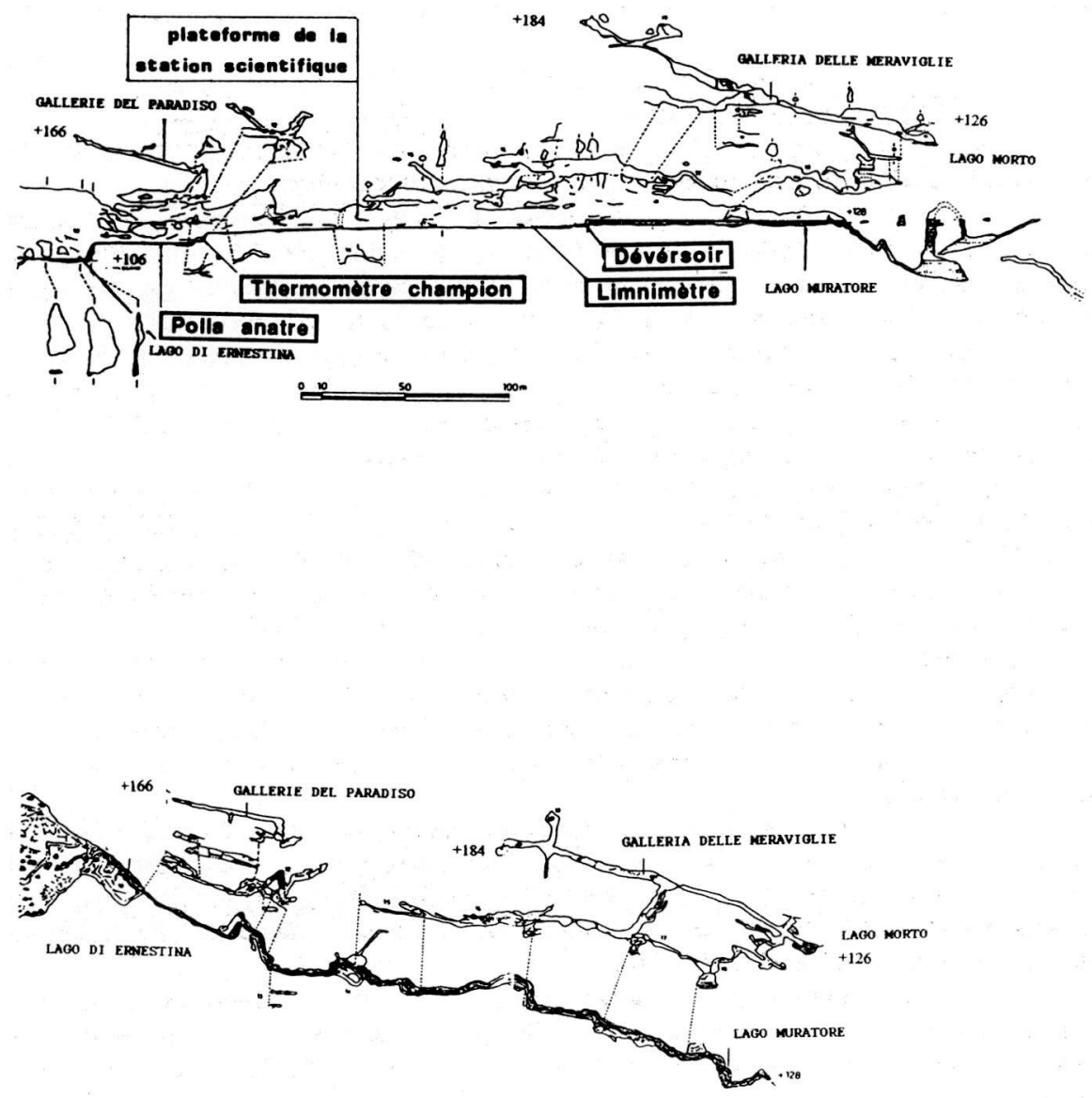

Fig. 2 - Plan de la "Grotta di Bossea": zone supérieure. 


\section{INTRODUCTION}

La "Grotta di Bossea" s'ouvre à 836 m d'altitude, à l'endroit du même nom, sur la gauche hydrographique du torrent Corsaglia (cfr. Carta d'Italia 1:25.000 dell'I.G.M., foglio 91, I SE). Elle a un développement de $2.800 \mathrm{~m}$ environ et présente un dénivellement global de $199 \mathrm{~m}$. Elle est parcourue par un torrent caractérisé par des crues élevées (jusqu'à $1200 \mathrm{l} / \mathrm{s}$ )

\section{CARACTERISTIQUES DE LA CAVITE}

La grotte est divisée en deux parties bien différentes du point de vue morphologique: zone inférieure et zone supérieure (Fig. 1 et 2).

La partie inférieure va de l'entrée au "Lago di Ernestina". S'étendant sur un $\mathrm{km}$ environ, elle a un dénivellement ascendant de $120 \mathrm{~m}$. Elle est formée surtout par une suite de grandes salles, parmi lesquelles on distingue la avec ses dimensions de $100 \times 60 \times 45 \mathrm{~m}$ de haut. La verticalité de ses parois, les vastes plafonds entaillés à arêtes aigues, la présence de blocs gigantesques, les concrétions grandioses, les cascades du torrent donnent à cette partie de la grotte un aspect majestueux et pittoresque. C'est le secteur de la cavité aménagé pour la visite touristique.

La partie supérieure est constituée par le canyon du torrent, très haut et étroit, qui se développe horizontalement et par les galeries fossiles au-dessus, subhorizontales ou inclinées, ornées de concrétions spectaculaires.

La cavité possède une grande valeur esthétique et naturalistique à cause d'un caractéristique particulière: la pleine vitalité du processus spéléogénétique. Bossea se présente donc comme une vraie, parcourue par des eaux impétueuses, dans laquelle le processus de formation est encore en plein développement avec de grandioses phénomènes lithogénétiques.

Tout cela est lié au dynamisme et à l'activité chimique et physiques des eaux courantes.

Cette action stimulante des eaux, augmentée par le fort dénivellement de la partie inférieure de la cavité (partie aménagée), les remarquables effets scénographiques (cascades, rapides, petits lacs), avec les grandioses dimensions des salles et les belles concrétions calcaires, donnent à la grotte une considérable valeur esthétique.

La grotte présente en même temps de très intéressants aspects naturalistiques et scientifiques pour ce qui concerne l'hydrogéologie, la météorologie, la biologie et la paléontologie, qu'on étudie depuis plusieurs années.

Tous les phénomènes physiques et biologiques qui ont eu lieu dans le passé ou qui se vérifient à présent, y ont déterminé, en 1970, la création d'un laboratoire souterrain pour les recherches expérimentales dans les domaines susmentionnés: la "Stazione Scientifica di Bossea", créée et gérée par le Groupe Spéléologique Alpi Marittime du C.A.I. de Cuneo, en collaboration avec le "Dipartimento Georisorse e Territorio" et le "Dipartimento di Elettronica" du Politechnique de Turin.

Cette réalisation, destinée à des buts autonomes derecherche mais aussi étroitement liée au développement touristique de la cavité, sera 
analysée dans une brève relation particulière.

\section{L'INTERET HISTORIQUE}

La première exploration de la grotte remonte à 1850 , d'autres eurent lieu dans les années suivantes. En 1875, on atteignit la première fois la partie supérieure de la cavité en dépassant la cascade du "Lago di Ernestina". Devenue célèbre par sa beauté, son intérêt hydrologique et par les fossiles d'Ursus spelaeus, Bossea fut déjà à cette époque-là objet des études de géologues et naturalistes.

En 1874, elle fut aménagée pour la visite touristique et ouverte au public. La célèbrité de la grotte se répandit dans plusieurs régions d'Italie et pendant quelques dizaines d'années, elle fut visitée par un grand nombre de visiteurs. La gestion touristique a duré, avec des hauts et des bas, jusqu'à nos jours.

L'exploration de la cavité fut achevée, dans ses développements essentiels, seulement en 1948. Ensuite, on a cependant découvert d'autres galeries collatérales qui s'étendent dans l'ensemble pour un $\mathrm{km}$ environ. La recherche de nouvelles parties de la cavité continue encore. Plusieurs tentatives pour franchir le syphon terminal de la part du G.S.A.M., à partir des années ' 60 , n'ont pas eu de bons résultats même s'ils ont permis l'exploration d'une partie assez considérable du grand réseau de galeries submergées.

Bossea fut une des premières grottes italiennes aménagées pour la visite du public. Les explorations effectuées, les importantes découvertes paléontologiques, le précoce aménagement touristique eurent un grand retentissement et répandirent dans tout le Piémont la connaissance et l'appréciation du milieu souterrain et de la culture spéléologique qui caractérise la région, et qui a eu dans la seconde partie de ce siècle son plus grand développement.

\section{LA STATION SCIENTIFIQUE}

La "Stazione Scientifica di Bossea", destinée à l'étude expérimentale du milieu karstique souterrain, est divisée dans les sections biospéléologique et hydrologique.

La Station Biologique, installée en 1969 dans la partie inférieure de la cavité, est constituée d'un laboratoire équipé avec des terrariums, des acquariums et d'autres appareils qui permenttent des recherches non seulement systématiques mais aussi de caractère biologique et éthologique, sur les organismes de grotte.

La Station Hydrogéologique, progressivement équipée à partir de 1973 mais en fonction continue depuis 1982, est située dans la partie supérieure de la cavité, à 800 mètres environ de l'entrée. L'appareillage, disposé surtout dans le canyon du torrent, est formé d'appareils automatiques et manuels pour le relèvement continu ou périodique de nombreux paramètres 
hydrologiques et météorologiques.

L'activité est adressée surtout vers trois buts de recherche :

- l'analyse détaillée d'un échantillon aquifère karstique concernant l'identification du réseau d'alimentation, les modalités d'absorption et de débit des eaux, l'hydrographie générale du réseau, l'analyse des caractéristiques chimiques et physiques des eaux et la corrélation de ces paramètres avec les phénomènes météorologiques extérieurs.

Un des buts de l'étude est l'élaboration d'un modèle de système karstique de moyenne altitude, très précis et en même temps assez flexible pour être appliqué à des systèmes karstiques analogues.

Tout cela pourra permettre de définir des procédés de protection du sol et des eaux qui, en considérant les caractères spécifiques des différents milieux karstiques, préviennent et éliminent les pollutions chimiques et biologiques possibles, et la programmation d'une utilisation rationnelle des ressources hydrologiques disponibles:

- l'étude climatologique du milieu souterrain par rapport à la situation météorologique de l'extérieur et aux variations du régime de la circulation hydrologique de l'intérieur ;

- l'étude de procédés de relevé et d'interprétation des données et d'appareils de relèvement automatiques des paramètres hydrogéologiques, chimico-physique et météorologiques du système souterrain. Les résultats de cette recherche permettront de déterminer les appareils et les systèmes de relèvement et d'étude des données pour des stations de mesure simplifiées pour le contrôle d'autres systèmes ou milieux karstiques.

Avec les recherches effectuées jusqu'à ce moment, on a la disponibilité de séries chronologiques s'étendant sur plusieurs années d'observation et de données, relevées de façon continue ou périodique. Ces séries sont quelquefois incomplètes ou irrégulières pour les difficultés opérationnelles interposées d'un appareillage n'étant pas encore adéquat et des conditions ambiantes défavorables. En plus, souvent les relevés périodiques ne permettent pas une analyse complète des différents paramètres et en limitent les possibilités de corrélation.

Cependant, on a pu acquérir un ensemble d'informations qui permettent l'interprétation des causes des phénomènes à l'étude et la connaissance de la modalité de la circulation hydrologique souterraine et du processus karstique. Les relevés météorologiques, jusqu' à ce moment pas encore continus, ont permis de déterminer certains aspects essentiels climatologiques de la cavité. Les résultats obtenus sont rapportés dans les "Atti della Stazione Scientifica di Bossea", publiés depuis peu.

La "Stazione Scientifica" a aussi un rôle important dans la valorisation ambiante, dans la sauvegarde écologique et dans l'information des visiteurs, réalisé entre autres dans la consultation aux projets de valorisation touristique, dans les stages de formation des guides, dans la préparation 
du matériel de documentation (guide monographique, brochures, etc.).

Le développement de l'activité de recherche prévoit, à court terme, l'acquisition d'appareils appropriés aux exigences opérationnelles, le contrôle aux instruments des nouvelles parties du réseau karstique et l'automatisation totale du relèvement et de l'élaboration des paramètres les plus importants. Cela permettra une disponibilité rapide d'un ensemble de données plus complet et continu et un considérable enrichissement des connaissances.

De cela résultera encore un emploi plus systématique des données pour une meilleure utilisation touristique de la cavité. L'utilisation de ces données concernera la sauvegarde du milieu, la sécurité des usagers et l'information culturelle. Donc, au contrôle constant de la situation du milieu, à l'organisatin rationnelle du flux touristique, au maintien des meilleures conditions de visite, on adjoindra une information continue des visiteurs en temps réel, par l'entremise d'un vidéo-computer, sur les phénomènes physiques et biologiques en cours dans la grotte.

\section{LES POTENTIALITES DE VALORISATION TOURISTIQUE}

La Grotta di Bossea présente donc ces principaux facteurs d'intérêt:

- vitalité du processus spéléologénétique et des phénomènes physiques et chimiques annexes;

- grande richesse d'eaux courantes;

- grandes dimensions des salles avec des paysages pittoresques et escarpés;

- remarquable valeur naturalistique et scientifique;

- existance d'une installation scientifique à l'intérieur;

- conséquante disponibilité de vastes connaissances pour la sauvegarde ambiante, la sécurité et l'information du visiteur.

Ces caractéristiques déterminent une grande potentialité d'utilisation touristique de la grotte soit esthétique soit culturelle. Mais au moment actuel, une telle potentialité est exploitée d'une façon limitée à cause de l'inadéquation des structures de visite, la carence des installations externes complémentaires, de $\mathrm{l}$ ' absence de matériel de documentation et d'information culturelle du public, de l' insuffisante capacité réceptive de l'environnement, de l'insuffisante publicité de la grotte et en général des difficultés financières limitant fortement les possibilités opérationnelles aussi bien dans le domaine structural qu'en ce qui concerne la gestion.

Pourtant la valorisation touristique de la Grotte de Bossea est, au moment actuel, inférieure aux possibilités réelles. Il est donc évident que la grotte est tout à fait digne d'être relancée au point de vue touristique et culturel pour y diriger un nombre convenable de visiteurs et produire pour la zone tous les avantages qui dérivent d'une forte augmentation des présences touristiques. 
Pour cela, la Commune de Frabosa Soprana, avec la consultation de la "Stazione Scientifica di Bossea", a préparé un projet global de valorisation et développement de la cavité, pour lequel a été demandé le financement de la aux termes des lois en vigueur.

\section{LES ORIENTATIONS EN PROJET}

Point fondamental dans la détermination des buts du projet est la recherche d'un programme, même si ambitieux, organisé et global et divisé seulement en deux phases: en effet, on a l'intention d'affronter dans le même temps et sur toute l'étendue du secteur touristique, l'ensemble des carences de l'aménagement.

De l'analyse de la condition actuelle, on relève que des interventions locales, partielles et irrégulières seraient insuffisantes à résoudre complètement les problèmes à caractère fonctionnel, de sécurité et d'utilisation du parcours de visite. Le caractère global qui doit caractériser l'intervention exige qu'elle s'étende aussi aux travaux nécessaires à la valorisation de la grotte et aux potentialités externes de réception.

Cela détermine donc une proposition de temps de réalisation divisée seulement en deux phases, dans lesquelles les interventions s'adressent à améliorer les conditions de visite selon les buts susmentionnés. Cette subdivision permet en outre de grouper dans chacune d'elles tous les travaux qui, même si concernant des secteurs technologiques distincts, sont étroitement en corrélation ou conséquents chronologiquement.

Dans la première phase, l'objet de l'intervention sera surtout l'intérieur de la cavité, tandis qu'on renvoie à la deuxième la solution des problèmes de l'accès et de carence de services à l'extérieur, ainsi que l'achèvement des oeuvres d'amélioration à l'intérieur.

A l'intérieur de la cavité, on réalisera des itinéraires alternatifs pour décongestionner le flux touristique dans les périodes de pointe et pour permettre la vue de parties de grotte actuellement inconnues au public. Ces itinéraires, alternatifs pendant la période des travaux, seront additionnels à la fin des travaux et consentiront en outre d'éviter l'élargissement du siège routier, en le limitant seulement aux tronçons à double sens de marche.

Les itinéraires supplémentaires sont prévus de toute façon dans les zones les plus vastes de la cavité (la Sala del Baldacchino, la Sala dell'Orso, la Sala Garelli), là où les grandes dimensions des salles permettent le camouflage des structures en réduisant le risque d'impact visuel de celles-ci.

L'utilisation est ensuite ultérieurement améliorée en procédant à l'agrandissement des petites places actuellement existantes mais de dimensions insuffisantes et à la création de nouvelles aires de stationnement et d'écoute des informations des guides, dans les points panoramiques les plus importants et où il y aura le croisement des groupes qui marchent dans la direction opposée. La typologie à adopter sera préférablement celle se rapportant aux structures à balcon permettant d'éviter la vision frontale des structures de soutien. 
C'est encore une action sur tous les fronts celle qu'on a programmé sur l'installationde l'électricité: on a prévu la substitution des appareillages dégradés et donc sans sécurité, mais aussi le camouflage des parties nouvelles pour éviter des contrastes et des éblouissements qui détournent le regard ou qui donnent de la gêne; on étendra l'installation de l'électricité aux itinéraires alternatifs mais on posera aussi des phares sous les eaux pour exalter la beauté extraordinaire de l'hydrographie de la cavité.

Puisque c'est là un des points de mérite de la grotte, on a décidé d'ajouter ces oeuvres à la première phase.

On imperméabilisera les lits des bassins qui existent encore mais sans alimentation du torrent et on rétablira la circulation des eaux dans quelques parties du vieux lit du torrent avec la remise en activité de rapides et cascades. Le bassin de la "Sala dell'Orso", presque toujours peu visible étant caché sous un rocher, sera agrandi pour être vu avec n'importe quel débit du torrent.

La première phase du programme comprend l'amélioration des possibilités de recherche de la dont on reconnaît le rôle central, indispensable à la protection du milieu et du visiteur, et la qualification du niveau culturel et scientifique. On prévoit donc d'en augmenter les appareillages et les structures de base pour étendre les connaissances du système karstique, au service de l'utilisation touristique de la cavité.

Mais si ces travaux améliorent les conditions de parcours et permettent une augmentation du flux touristique, un ultérieur essor devra avoir la valorisation et surtout la dotation de services à l'extérieur.

Pour ce qui concerne l'accès à la grotte, dans cette seconde phase on prévoit le changement de la rampe pour éviter l'interruption des visites ou au moins une viabilité difficile sur le chemin actuel. D'autres objectifs aussi ont conseillés cette solution: le nouveau point de départ est central par rapport aux parkings actuels (de toute façon à agrandir), on a ainsi tout le parcours sur territoire communal, on peut "reformer" le vert interrompu par les lacets actuels de la rampe et former ainsi un écran devant la nouvelle. Les derniers lacets seront utilisés comme plate-forme pour la réalisation d'installations sanitaires et d'emplacements pour l'attente (l'un d'eux sera couvert).

Au niveau de la route on prévoit une structure polyvalente, au bord du parking, où il y aura des guichets, des installations sanitaires pour les visiteurs et le personnel, une salle pour le musée et les projections et pour abriter les groupes par mauvais temps.

Même la rampe en projet qui, avec une différence de niveau de $17 \mathrm{~m}$, relie la route et l'entrée, sera couverte et formera un parcours équipé pour la documentation préliminaire du touriste avec l'installation de tableaux explicatifs.

Le programme se complète, à l'intérieur, avec l'élargissement des autres parties du parcours (sauf le couloir d'entrée où l'on préfère l'installation d'un feu) avec des structures appropriées pour la section biologique de la et des terrariums qui pourront en partie être transférés dans la dont on prévoit la réorganisation fonctionnelle comme musée à l'intérieur. 


\section{CHOIX OPERATIONNELS ET MODALITES EXECUTIVES}

Si d'un côté il est nécessaire, pour la première chose, d'éliminer les causes du danger, de l'autre on doit rendre plus facile le parcours à l'intérieur et l'accès à la grotte avec une particulière attention aux normatives concernant l'abolition des barrières architectoniques. La régularisation del'escalier actuel et des nouvelles parties en projet devront tenircompte des rapports entre la contre-marche et l'emmarchement, fixés de et les mains courantes des rampes seront doubles (l'une à $\mathrm{cm} 60$ de haut) pour permettre l'appui même aux enfants.

L'introduction de tout ces éléments fonctionnels, de même que ceux relatifs à décoration ou technologies, est, dans ce milieu particulier, extrêmement délicat: on doit éviter un désagréable impact ambiant mais aussi on doit tenir compte de la nature périssable des matériels dans un milieu très humide même si non pollué et des difficultés de transport.

Pour cela on a choisi des éléments préfabriqués légers, faciles à réunir avec des processus qu'on peut diversifier selon les nécessités d'encrage. Les structures portantes seront préfabriquées en usine et montées sur place. Les escaliers auront des marches en grilles galvanisées antidérapantes avec des plaques latérales pour les fixer aux longerons par des boulons inox et d'un profilé antérieur en renfort et antidérapant.

Avec ces marches qui ne retiennent ni eau ni terre, on augmentera le niveau de sécurité du parcours, parfois glissant; le degré du bruit du métal lors du piétinement sera couvert par le bruit caractéristique du torrent. Les passerelles aussi seront réalisées avec les mêmes grilles à mailles serrées mais en même temps légères et lumineuses. Pour les mains courantes, on fera usage de tuyaux Mannesmann pour installations hydrauliques qui en plus d'être en fer galvanisé comme les autres structures, sont reliés entre eux à l'aide de normaux manchons filetés, par des opérations faciles à réaliser sur place. La continuité de la main courante, tout à fait indispensable pour des raisons de sécurité, permettra d'employer les tuyaux comme conduite de l'eau pour le lavage de la cavité. naturels locaux (pierre, bois) même si utilisés en typologie moderne et rapprochés des éléments plus actuels en verre et ciment armé à vue.

Comme dans les centres historiques urbains ou dans les villages de campagne ou de montagne de remarquable valeur artistique, le résultat des nouvelles introductions est dû surtout à la sensibilité de l'architecte puisque on ne peut avoir des règles univoques. Dans ce cas, on a l'appui de la critique compétente de la qui s'ajoutera à l'avis des organisations préposées à la sauvegarde du milieu.

Par analogie avec les interventions citées au-dessus, les choix des matériels et des typologies sont celles qui exaltent, avec un contraste délicat, les beautés existantes et s'harmonisent sans prévarique. 


\section{CONSIDERATIONS FINALES}

Par rapport aux potentialités caractéristiques de la Grotta di Bossea, si mises en valeur, on peut supposer, une augmentation du flux touristique de 35-40.000 présences annuelles à 110-120.000. On a déjà eu un certain accroissement, mais le caractère concret de cet objectif est lié non seulement aux oeuvres en projet mais aussi à une forte activité promotionnelle pour attirer l'intérêt des touristes au-delà des limites des régions piémontaises et limitrophes (les visiteurs proviennent surtout du Piémont, du Val d'Aoste, de la Ligurie et de la Lombardie).

En plus, la zone sur laquelle l'intervention pourra avoir une rechute économique est bien plus grand que celle locale, sauf évidemment les activités commerciales les plus proches de la grotte: outre que consolider la mise en ordre socio-économique globale de la zone (le maintien du peuplement, sauvegarde ambiante, les soins de l'environnement), les avantages de l'augmentation touristique auront un écho régional. 